Textures and Microstructures, Vol. 30, pp. 55-70 Reprints available directly from the publisher Photocopying permitted by license only
(C) 1997 OPA (Overseas Publishers Association) Amsterdam B.V. Published in The Netherlands under license by Gordon and Breach Science Publishers

\title{
FEATURES OF THE PHASE TRANSFORMATIONS IN SHEETS, TUBES AND WELDING SEAMS OF THE ALLOY $\mathrm{Zr}-2.5 \% \mathrm{Nb}$
}

\author{
M. ISAENKOVA and Yu. PERLOVICH* \\ Moscow Engineering Physics Institute, Kashirskoe shosse 31, \\ 115409 Moscow, Russia
}

(Received 20 February 1997)

\begin{abstract}
By use of X-ray texture analysis characteristic features of phase transformations in sheets, tubes and welding seams of the $\mathrm{Zr}-2.5 \% \mathrm{Nb}$ alloy were studied. Main attention was given to inhomogeneity of phase transformation development, connected with inhomogeneous distribution of strain hardening in grains with different crystallographic orientations. The typical manifestation of phase transformation inhomogeneity in cold-rolled sheets is a shift of the initial texture maximum to the region of increased strain hardening. In channel tubes in consequence of phase transformations $\alpha-\beta-\alpha$ the texture component $\{11 \overline{2} 0\}\langle 10 \overline{1} 0\rangle$ gains, testifying that tangential stresses favour corresponding alignment of basal normals accompanied by absorption of grains with other orientations. Competition between recrystallization and phase transformation in conditions of heating was considered. As strain hardening increases, recrystallization prior to phase transformation becomes more probable, being predominant within $\alpha$-grains of some orientations. Parallel development of recrystallization and phase transformation in the zone of thermal influence by arc welding of rolled sheets was considered. It was shown that, as the distance from the melting zone decreases, different combinations of these processes realize.
\end{abstract}

Keywords: Phase transformation; $\mathrm{Zr}-\mathrm{Nb}$ alloy; Burgers orientation relationship; recrystallization

\section{INTRODUCTION}

Usage of zirconium alloys in atomic energetics and chemical engineering requires detailed investigation of structure formation processes in these alloys under their treatment. Some technological procedures

\footnotetext{
* Corresponding author.
} 
involve heating of semifinished items up to a temperature of the phase transformation (PT) $\alpha-\beta$ as well as their subsequent cooling with PT $\beta-\alpha$, and then, as a result, both initial structure and texture can change radically. Forging, pressing, hot rolling are procedures aimed for deformation of a massive slab which are inevitably connected with phase transformations. By welding, a wide spectrum of local conditions take place, and in a definite zone the material experiences PT, causing there a sharp structure inhomogeneity with various negative consequences for operational properties. In some cases a special heat treatment with final quenching of the products is used to raise grain dispersity and to attain corresponding strengthening. In addition, concurrent texture scattering decreases radiation growth and creep anisotropy of treated channel tubes. Regularities of these processes originate from PT features, typical for used zirconium alloys.

The given work is devoted to description of some characteristic features of PT development in products of the commercial $\mathrm{Zr}-2.5 \% \mathrm{Nb}$ alloy on the basis of X-ray data. At room temperature in equilibrium state this alloy consists predominantly of the $\alpha$-phase with HCP crystalline lattice and includes also separate particles or interlayers of the $\beta$-phase with BCC-lattice. As a rule, the $\mathrm{X}$-ray method allows to register in the $\mathrm{Zr}-2.5 \% \mathrm{Nb}$ alloy only traces of $\beta-\mathrm{Zr}$, and in any case its content, by $\mathrm{X}$-ray data, does not exceed a few volume percents. Sometimes transmission electron microscopy (TEM) estimates the content of $\beta-\mathrm{Zr}$ in the $\mathrm{Zr}-2.5 \% \mathrm{Nb}$ alloy somewhat higher. This difference can be connected with the local character of TEM data as well as with an anisotropic sensitivity of X-ray diffractometry relative to particles of secondary phases in the form of thin plates or needles. According to the state diagram $\mathrm{Zr}-\mathrm{Nb}, \mathrm{PT} \alpha-\beta$ in the alloy $\mathrm{Zr}-2.5 \% \mathrm{Nb}$ starts at $610^{\circ} \mathrm{C}$ by attainment of the monotectoid line and is finished at $840^{\circ} \mathrm{C}$ by attainment of the boundary between the $(\alpha+\beta)$ - and $\beta$-regions (Douglass, 1971).

As a result of quenching in the alloy so-named $\alpha^{\prime}$-phase forms, which has a distorted $\alpha$-lattice and is supersaturated with $\mathrm{Nb}$. Annealing converts the nonequilibrium $\alpha^{\prime}$-phase into the equilibrium $\alpha$-phase. But this process cannot be considered as PT in the conventional sense, because the type of lattice and grain orientations remain unchanged, while the X-ray evidence of structure changes consists only in narrowing of the X-ray lines. Therefore, while considering PT in the 
$\mathrm{Zr}-2.5 \% \mathrm{Nb}$ alloy on the basis of X-ray data, we do not distinguish $\alpha^{\prime}-$ and $\alpha$-phases, though a dependence of $\alpha$-lattice perfection on the cooling rate is taken into account.

Physical studies in the field of PT are informative only on condition that X-ray texture analysis is used, since only this method allows to distinguish grains with different prehistories by their crystallographic orientations and to reveal mechanisms of structure formation, acting in the course of PT. Namely, this circumstance is responsible for the most attention to texture data in the given work.

\section{BASIC FACTS AND TOPICAL CONCEPTIONS}

The literature on the subject is quite extensive. In particular, different aspects of PT in zirconium alloys are considered in general monographs (Douglass, 1971; Tenckhoff, 1980; Zaymovskiy et al., 1981). It is known that PT $\alpha-\beta-\alpha$ obeys the Burgers orientation relationship:

$$
\{0001\}_{\alpha}\left\|\{011\}_{\beta}, \quad\langle 11 \overline{2} 0\rangle_{\alpha}\right\|\langle 111\rangle_{\beta} .
$$

Multiplying of initial orientations in consequence of PT $\alpha-\beta-\alpha$ was analysed by Cheadle and Ells (1966). By absence of variant selection 35 new orientations of the basal plane arise in addition to the initial one; while taking into account mutual coincidence of some orientations, their total number decreases down to 24 . Since in reality we deal with textured polycrystals of zirconium alloys instead of single crystals, after PT $\alpha-\beta-\alpha$ the resulting distribution of basal normals on the stereographic projection consists of overlapping texture maxima rather than of separate points. Therefore some new orientations of the 24 abovementioned, being close to each other, form common maxima. Thus, PT complicates an initial texture, multiplying its maxima in a definite way, though the resulting direct pole figure (DPF) (0001) of the treated sample contains a lesser number of separate maxima than the Burgers relationship predicts.

According to the popular point of view (Tenckhoff, 1980), recrystallization of $\alpha-\mathrm{Zr}$ transforms its rolling texture by way of $30^{\circ}$ rotation around the basal normals without essential changes of DPF (0001). As a result, after PT $\alpha-\beta-\alpha$ the arrangement of texture maxima 
on DPF (0001) for the case of previously recrystallized $\alpha-\mathrm{Zr}$ is other than for the case of PT in the rolled state. This difference allows to reveal, by the final distribution of basal normals, the initial state of the treated sample, having been exposed to PT. However, under certain circumstances PT and recrystallization can be in mutual competition, and therefore recrystallization can develop during heating of a deformed sample up to PT temperature. In particular, it was established that during heating to the $\beta$-phase rolled Zircaloy- 2 recrystallizes, while the $\mathrm{Zr}-2.5 \% \mathrm{Nb}$ alloy does not (Cheadle and Ells, 1966). Evidently, movement of both intergranular and interphase boundaries is governed by similar regularities, but its dependences on lattice distortion, strain hardening distribution and mutual misorientation of neighbouring grains are somewhat different. Therefore, variation of local conditions can favour or restrain recrystallization of the $\alpha$-phase before PT $\alpha-\beta$. The most favourable factor for recrystallization during heating to the $\beta$-phase seems to be high strain hardening, increasing near intermetallic particles in Zircaloy-2.

In recent years, owing to progress of X-ray diffractometry, new data were obtained, refining significantly the physical concepts of deformation and texture formation processes as well as of structure inhomogeneity induced by any technological treatment of zirconium alloys. In this connection a repeat cycle of X-ray investigations in the field of PT development with reference to zirconium alloys is necessary, taking into account the following new aspects.

(1) Experimental methods were elaborated to compare dispersity and lattice distortion in grains with different crystallographic orientations (Perlovich, 1983); it was shown, by use of such methods, that structure inhomogeneity of treated metal materials is controlled by their texture and that at the periphery of texture maxima grain dispersity and lattice distortion are higher than in central regions of these maxima (Perlovich, 1994). In $\alpha$-Zr various mechanisms of plastic deformation are acting depending on grain orientations: basal, prismatic and pyramidal slip, three systems of twinning; each local combination of these mechanisms results in the characteristic substructure and strain hardening (Isaenkova, Perlovich and Kapliy, 1994).

(2) Kinetics of texture formation in $\alpha$-Zr was studied, beginning from different typical initial states of the material. New variations of rolling texture were described with a parallel analysis of acting mechanisms of 
plastic deformation. Stability of grain orientations is conditioned by equilibrium of different mechanisms in the course of deformation. Thus, by cold rolling of a textureless slab, the pair of texture components $(0001) \pm 10^{\circ}-20^{\circ} \mathrm{ND}-\mathrm{RD}\langle 11 \overline{2} \mathrm{~L}\rangle$ forms, being quasi-stable at intermediate deformation degrees due to the joint action of basal and pyramidal slip (Isaenkova and Perlovich, 1987). Stability of the final texture components $(0001) \pm 20^{\circ}-40^{\circ} \mathrm{ND}-\mathrm{TD}\langle 10 \overline{1} 0\rangle$ is maintained by the joint action of prismatic and basal slip. While the texture development by PT $\alpha-\beta-\alpha$, for the case of the final rolling texture, was known earlier (Cheadle and Ells, 1966), the PT texture, corresponding to the quasi-stable intermediate rolling texture, is investigated in the given work.

(3) In works after Isaenkova et al. $(1988,1989)$ the detailed analysis of recrystallization in sheets and tubes of zirconium alloys was performed. Contrary to Douglass (1971) and Tenckhoff (1980), it was established that, in reality, grain reorientation by recrystallization cannot be described as only $30^{\circ}$-rotation around a motionless basal normal. As a rule, recrystallization of a rolled product involves several stages, corresponding to mismatched recrystallization of different texture components. In addition to the known rotation around the basal normals, an important feature of recrystallization in $\alpha-\mathrm{Zr}$ was revealed. This is some shift of the maxima on DPF (0001), testifying about a redistribution of the basal normals in the course of recrystallization. A new position of the texture maximum on DPF (0001) for a recrystallized $\alpha-\mathrm{Zr}$ is at the slope of the initial maximum, belonging to the rolling texture. According to the general regularity, at slopes of texture maxima strain hardening increases, and this is a reason for inhomogeneous nucleation and growth of recrystallized grains namely here. A question arises, whether PT $\alpha-\beta$ is sensitive to the distribution of strain hardening in $\alpha-\mathrm{Zr}$, as it was observed for recrystallization.

\section{MATERIAL TREATMENT AND INVESTIGATION TECHNIQUE}

Samples for investigation were cut from sheets and tubes of the $\mathrm{Zr}-2.5 \% \mathrm{Nb}$ alloy, cold-rolled by different deformation degrees. In order to induce PT $\alpha-\beta-\alpha$, samples were subjected to heat treatment in dynamic vacuum, including heating up to $950^{\circ} \mathrm{C}$ for $0.25 \mathrm{~h}$, holding at 
this temperature during $0.5 \mathrm{~h}$ and subsequent cooling with an evacuated envelope in air. For X-ray study the diffractometer DRON-3M and $\mathrm{Cu} \mathrm{K}_{\alpha}$-radiation was used. Texture measurements were carried out by the standard method (Borodkina and Spector, 1981), using the automatical texture unit under computer control. For constructing of a full DPF, three mutually perpendicular sections of a sheet/tube were investigated to obtain partial pole figures for their following sewing together. In some cases a procedure of extrapolation was applied for reconstruction of the periphery region of DPF.

The investigated welded joints were produced by argon-arc welding of a cold-rolled sheet of $\mathrm{Zr}-2.5 \% \mathrm{Nb}$ alloy, using a non-expendable tungsten electrode with a motion velocity of $60 \mathrm{~m} / \mathrm{h}$. The welding direction (WD) was perpendicular to the rolling direction (RD). Texture inhomogeneity near the welding seam, connected with different heating conditions at neighbouring regions, was studied layer by layer depending on the layer distance from the centre line of the seam.

\section{RESULTS AND DISCUSSION}

Obtained experimental results are presented in the following sections according to different aspects of interest.

\subsection{Inhomogeneity of PT in Cold-rolled Sheets}

If PT $\alpha-\beta-\alpha$ develops homogeneously in all $\alpha$-grains of the sample, the final texture of the $\alpha$-phase would contain, besides new maxima, also those maxima which are coincident precisely with the corresponding maxima of the initial texture. Any shift of such maximum after PT with respect to its original position would testify that, in the course of PT, some grains absorb partially their neighbours, having somewhat different orientations. Just, this situation is typical for PT in rolled sheets and tubes of the $\mathrm{Zr}-2.5 \% \mathrm{Nb}$ alloy.

Figure 1 shows a PT effect on the distribution of basal normals in sheets, rolled up to formation of the intermediate quasi-stable texture with the main components $(0001) \pm 12^{\circ} \mathrm{ND}-\mathrm{RD}\langle 11 \overline{2} \mathrm{~L}\rangle$. The final stable rolling texture of $\alpha-\mathrm{Zr}$ can vary to some degree depending on repeated usage of intermediate annealings for strain hardening removal. Thus, Fig. 2 shows the formation of PT texture in a foil, 

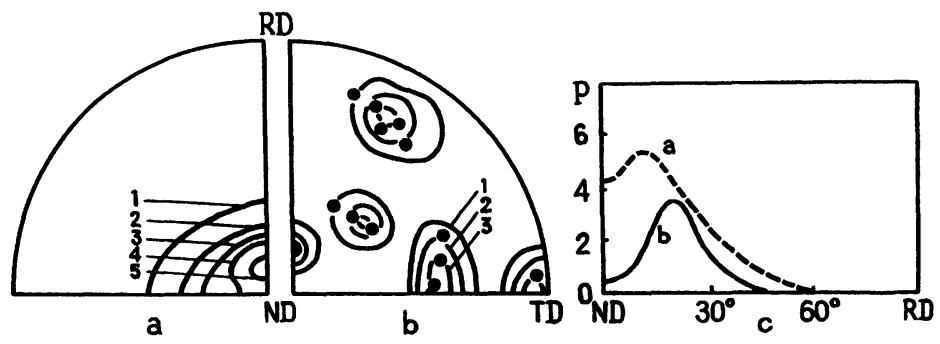

FIGURE 1 PT in the sheet with initial quasi-stable rolling texture, DPF (0001): (a) before PT; (b) after PT; (c) pole density distribution in the section ND-RD of DPF (a) and (b).

produced by rolling with intermediate annealings and very high integral deformation degree; the obtained rolling texture is rather perfect, while its main components $(0001) \pm 17^{\circ} \mathrm{ND}-\mathrm{TD}\langle 10 \overline{1} 0\rangle$ are at comparatively small distance from ND. In the case of cold rolling without intermediate annealings (Fig. 3) the sheet acquires high strain hardening and a scattered texture with the maximum at an angular distance of $26^{\circ}$ from ND. Shifts of the initial texture maxima in consequence of PT for all three cases is shown on diagrams (Figs. 1(c), 2(c), 3(c)), where distributions of pole density along the corresponding radius of DPF, both before and after PT, are drawn. The angular distance of the maximum top from ND increases by $9^{\circ}, 18^{\circ}$ and $5^{\circ}$, respectively.

Small black circles on Figs. 1(b) and 2(b) indicate all PT-orientations, appropriated to the shifted position of the maximum in the initial rolling texture; they are obtained assuming that the alloy was in the deformed state before PT. It can be seen that all new maxima of the final PT texture coincide precisely with several grouped circles. Apart from the shift of the initial maxima, a decrease of their angular extent testifies about an inhomogeneous character of PT.

The exhibited data confirm the validity of the above-suggested analogy between PT and recrystallization. In both cases, contributions of different orientations within the scattering of the initial texture are not mutually proportional in the final PT texture. At least two mechanisms can be responsible for this feature of the PT texture:

(A) Primarily PT begins in some grains with an increased energy of residual lattice distortions, whose orientation corresponds to the slope of the initial texture maximum on DPF (0001), as it was shown to be 

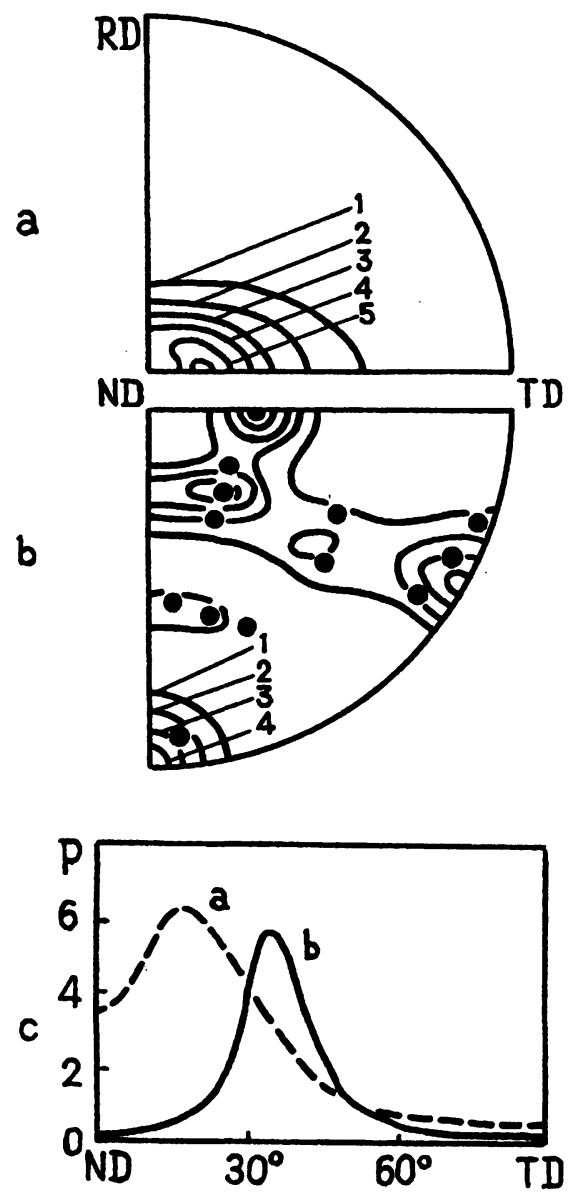

FIGURE 2 PT in the sheet with the initial stable texture obtained by rolling with intermediate annealings, DPF (0001): (a) before PT; (b) after PT; (c) pole density distribution in the section ND-TD of DPF (a) and (b).

typical for deformed metal materials (Perlovich, 1994). An interphase boundary, generated by local PT $\alpha-\beta$, moves into a neighbouring $\alpha$ grain with an orientation, differing somewhat from the initial orientation of the grain, where PT began at first. As a result, in this neighbouring $\alpha$-grain, PT $\alpha-\beta$ develops with violating the Burgers orientation relationship.

(B) Mutual absorption of grains, corresponding to different regions of the initial texture maximum, proceeds in the $\beta$-phase by motion of 

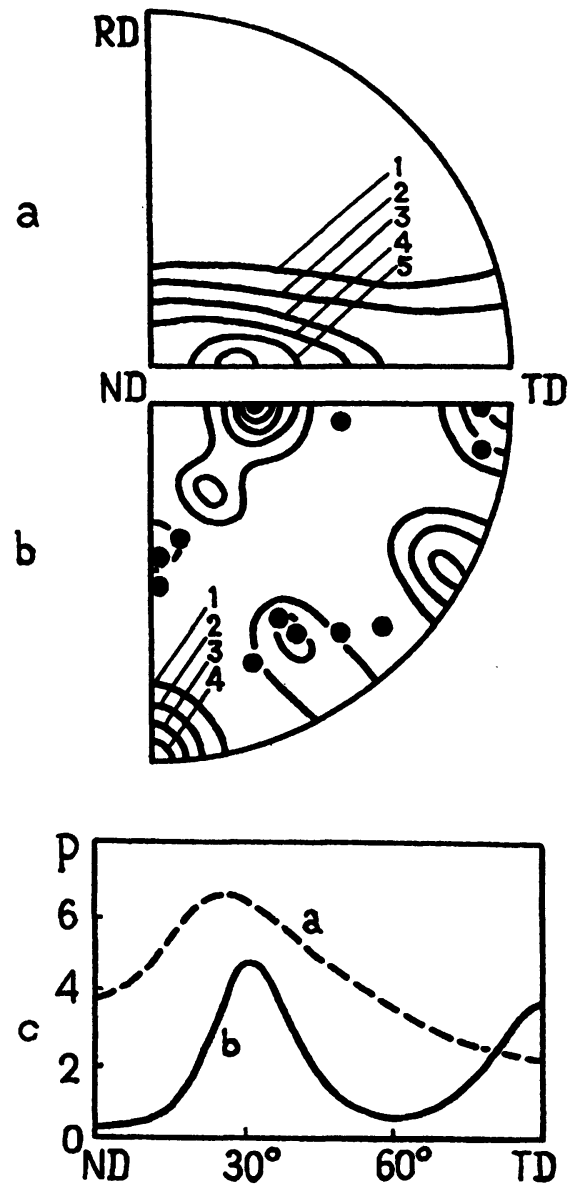

FIGURE 3 PT in the sheet with the initial stable texture obtained by rolling without intermediate annealings, DPF (0001): (a) before PT; (b) after PT; (c) pole density distribution in the section ND-TD of DPF (a) and (b).

intergranular boundaries. In each grain, regardless of its lattice distortion, PT follows the same orientation relationship, and only afterwards, in the course of holding at $950^{\circ} \mathrm{C}$, privileged growth of some $\beta$ grains takes place, resulting in a shift of maxima in the final PT texture.

Definite evidences exist that the mechanism $\mathrm{A}$ is more probable, though, in reality, both mechanisms could be activated. 


\subsection{Competition between Recrystallization and PT}

It is known that the tendency for recrystallization increases as strain hardening increases. Taking into account an inhomogeneous character of strain hardening distribution in metal materials with a developed rolling texture, we must expect that the local tendency to recrystallization would depend on grain orientation. With reference to $\alpha-\mathrm{Zr}$, this was shown experimentally in the above-mentioned works (Isaenkova et al., 1988; 1989) for cases of recrystallization annealings. In the present work inhomogeneous recrystallization was observed as an alternative to PT $\alpha-\beta$ by heating of the cold-rolled sheet with a high level of strain hardening up to $950^{\circ} \mathrm{C}$. This case is illustrated by Fig. 3 .

Analysis shows that the final texture after heat treatment (Fig. 3(b)) contains two sets of maxima, corresponding to PT from both rolled and recrystallized states. Black circles indicate here ideal orientations, obtained by "multiplication" of the shifted initial maximum assuming that, by the time of PT, $\alpha-\mathrm{Zr}$ was already recrystallized. In particular, as a result of PT $\alpha-\beta-\alpha$ in recrystallized grains, a new texture component $\{10 \overline{1} 0\}\langle 11 \overline{2} 0\rangle$ arises, having its maximum on DPF (0001) at TD. Other maxima of the same set are present at the predicted regions also, confirming additionally the fact of preliminary recrystallization in the course of heating to the $\beta$-phase. At the same time, maxima, originating from deformed $\alpha$-grains by PT, became noticeably weaker than in the case of PT without preliminary recrystallization (compare Figs. 2(b) and 3(b)). The rise of the new maximum at TD, due to recrystallization before PT, is seen also on Fig. 3(c), being in a sharp contrast with Fig. 2(c). Thus, we observe here partial recrystallization of the $\alpha$ phase before PT, though the heating rate was the same as in other cases.

Hence, the quoted statement about absence of recrystallization before PT time in the rolled $\mathrm{Zr}-2.5 \% \mathrm{Nb}$ alloy (Cheadle and Ells, 1966) is incorrect generally, because our results testify that, in some cases, the processes of PT $\alpha-\beta$ and recrystallization are competitive. The real situation in the deformed textured $\alpha$-phase requires a description similar to the case of a composite; therefore it would be correct to state a possibility of different outcomes from the competition between recrystallization and PT $\alpha-\beta$ depending on deformation degree, grain orientation, heating rate, and so on. Inhomogeneous development of the considered processes, as well as their mutual competition, correspond apparently to the most general case. 


\subsection{Features of PT Development in Tubes of $\mathrm{Zr}-2.5 \% \mathrm{Nb}$ Alloy}

A distinctive property of channel tubes, as compared with sheets of the same alloy, is the inevitable presence of significant tangential stresses. As a result of these stresses, both the rolling texture and the PT texture in channel tubes differ from those in sheets. In the course of cold rolling, in a tube a texture forms, containing usually, alongside with components $(0001) \pm 30^{\circ}-40^{\circ} \mathrm{ND}-\mathrm{TD}\langle 10 \overline{1} 0\rangle$, an intense component $\{11 \overline{2} 0\}\langle 10 \overline{1} 0\rangle$. In DPF (0001) the corresponding texture maximum is located at TD (Figs. 4(a) and (c)). Thus, the rolling texture of the tube may be considered as consisting of three main components, though their spread ranges overlap mutually.

In order to suppress recrystallization processes during PT heat treatment, the tube was preannealed at $350^{\circ} \mathrm{C}$. This annealing did not change the initial texture of the tube, but caused recovery and a decrease of strain hardening. As a result, structure inhomogeneity of grains with different orientations became less pronounced. In Fig. 4(b) the texture is shown, which developed in the tube by PT $\alpha-\beta-\alpha$. It has a complex character, understandable if multiplication of orientations would be considered for two main components of the initial rolling texture. White circles indicate new orientations produced by PT from the tangential texture component and black circles the orientations produced from the components $(0001) \pm 35^{\circ} \mathrm{ND}-\mathrm{TD}\langle 10 \overline{1} 0\rangle$. The genesis of all observed maxima becomes clear by their arrangement. Comparison of Figs. 3(b) and 4(b) testifies, at a first glance, about some similarity of the two PT textures, but detailed analysis reveals definite differences, which show quite unambiguously the absence of recrystallization in the investigated tube contrary to the situation in the sheet.

Preliminary removal of strain hardening resulted in more homogeneous PT $\alpha-\beta$, being evident from significant scattering of the final texture. Under conditions of relatively homogeneous structure in the preannealed tube, mutual PT absorption of grains with different orientations is suppressed substantially. Nevertheless, beside some small shift of the initial texture maximum at a distance of $35^{\circ}$ from ND, a prominent manifestation of PT inhomogeneity in the tube can be seen. This is the rather sharp redistribution of pole density between the two main components of the initial texture in consequence of PT $\alpha-\beta-\alpha$ (Fig. 4(c)). The tangential texture component builds up at the cost of the components $(0001) \pm 35^{\circ} \mathrm{ND}-\mathrm{TD}\langle 10 \overline{1} 0\rangle$. Such preference seems to 

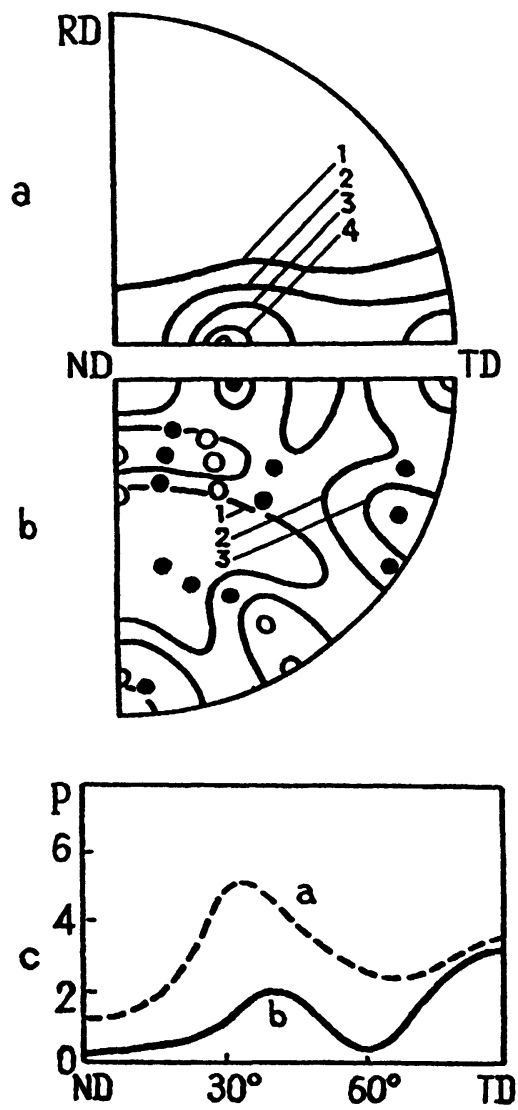

FIGURE 4 PT in the channel tube; DPF (0001): (a) before PT; (b) after PT; (c) pole density distribution in the section ND-TD of DPF (a) and (b).

be connected with the special character of the transverse direction in a tube, namely, along this direction the greatest stresses owing to the volume changes by PT are acting. Hence, a characteristic feature of PT development in a tube is an influence of tangential stresses on PT inhomogeneity.

\section{4. $P T$ in the Thermal Influence Zone by Welding}

Phase transformations in zirconium alloys by welding will be considered in detail in another paper. Here only some observations are presented, concerning the competition between recrystallization and 
PT $\alpha-\beta$ in the thermal influence zone (TIZ) with reference to the $\mathrm{Zr}-$ $2.5 \% \mathrm{Nb}$ alloy.

Arc welding is accompanied by a local heat treatment of the material in the vicinity of the welding seam. Parameters of a short-time thermal cycle, passing lengthwise TIZ parallel to the welding direction, are different for each longitudinal section of TIZ and depend on its distance from the central line of the seam. Layer-by-layer study of the texture within the TIZ gives an insight into inhomogeneous structure developed in this zone by welding. In Fig. 5(a) a schematic image of a welded joint is drawn; the melting zone is denoted by dense hatching and TIZ by thin hatching. Three longitudinal sections are shown within the TIZ, and for each section an arrow indicates the corresponding DPF (0001) obtained by X-ray diffractometric study just of this section. RD is brought into the centre of these DPF in contrast with the pole figures in the previous sections. Judging from the DPF in Fig. 5, different textures have formed in the shown sections of the TIZ depending on the distance from the seam. While in the most remote section the initial distribution of basal normals remains invariant, the textures of the two closer sections contain new components produced by PT. The pole figures for these sections differ in relationship of PT

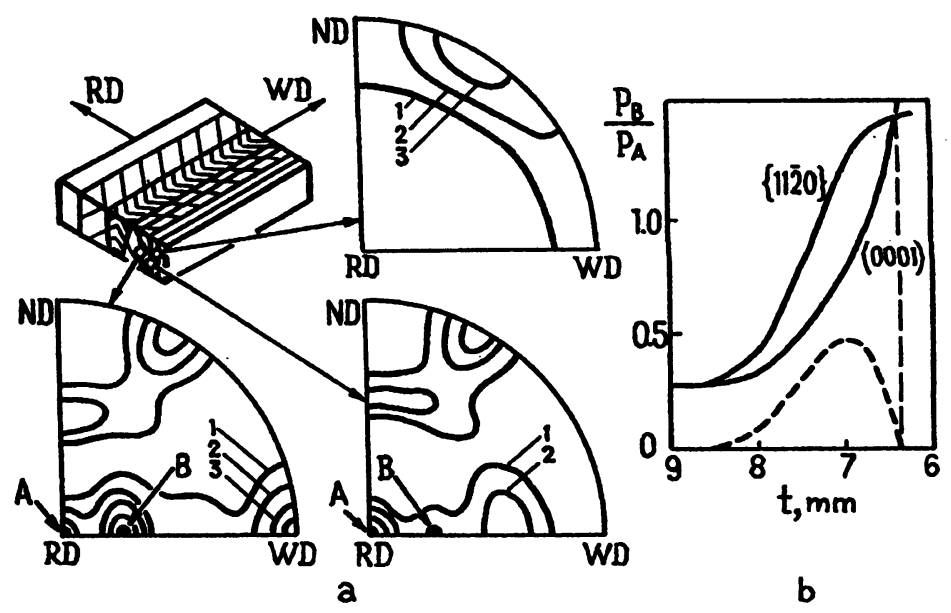

FIGURE 5 PT in the thermal influence zone by arc welding: (a) the schematic image of welding seam and DPF (0001) for its different sections; (b) quantitative treatment of DPF (see detailed explanation in the text). 
components, originating from deformed (A) and recrystallized (B) $\alpha$ grains. For each section DPF $\{11 \overline{2} 0\}$ was measured also.

A quantitative treatment of the obtained experimental data included the calculation of parameters, characterizing the relative contributions of both deformed and recrystallized components in the measured texture. In Fig. 5(b) the results of such treatment for 18 successive sections of the TIZ are presented. The upper curve, constructed by DPF $\{11 \overline{2} 0\}$, characterizes the relative fraction of recrystallized grains depending on the distance from the seam, irrespective of whether these grains experienced PT or did not. The following curve, constructed by DPF (0001), characterizes the relative fraction of recrystallized grains in volume covered by PT; it should be noted that in the general case this volume forms only a part of the investigated layer. The lower curve was obtained by subtraction of the (0001)-curve from the $\{11 \overline{2} 0\}$-curve. It characterizes the fraction, falling on grains, which recrystallized, but did not experience PT.

The presented curves testify unambigously that, by sufficient increase of the heating temperature, all grains experience recrystallization prior to PT $\alpha-\beta$. Thus, competition between recrystallization and PT, by a high rate of heating, results in an absolute predominance of primary recrystallization (variant 1), though, at some intermediate regimes of heat treatment, two other variants are possible: recrystallization of deformed $\alpha$-grains without their subsequent PT (variant 2) and PT of deformed $\alpha$-grains without their preliminary recrystallization (variant 3 ).

Thus, the most important feature of the processes in the thermal influence zone is their inhomogeneous character, particularly by intermediate regimes of heat treatment. While in some grains the variant 1 realizes, in other grains the variants 2 or 3 can take place. The concrete variant, actual for the given grains, depends in some complicated manner on their orientation. This dependence was revealed partially in previous sections, when considering shifts of initial texture maxima in the course of PT and recrystallization as a result of inhomogeneous strain hardening.

\section{CONCLUSIONS}

(1) Phase transformation $\alpha-\beta$ in cold-rolled sheets of $\mathrm{Zr}-2.5 \% \mathrm{Nb}$ alloy develops inhomogeneously, involving mutual absorption of 
grains with different orientations. The grains with orientations, displaced from the initial maximum of the rolling texture, experience PT first of all. According to the available data, these orientations belong to regions of increased strain hardening.

(2) By heating the sheet of the $\mathrm{Zr}-2.5 \% \mathrm{Nb}$ alloy a competition between recrystallization and PT $\alpha-\beta$ takes place in the case of sufficiently high strain hardening. As a result, an inhomogeneous structure forms, consisting of grains, which experienced PT both in the deformed and recrystallized states.

(3) The characteristic manifestation of the inhomogeneity of phase transformation in channel tubes of the $\mathrm{Zr}-2.5 \% \mathrm{Nb}$ alloy is a final relative gain of the tangential texture component $\{11 \overline{2} 0\}\langle 10 \overline{1} 0\rangle$ at the cost of the components $(0001) \pm 35^{\circ} \mathrm{ND}-\mathrm{TD}\langle 10 \overline{1} 0\rangle$, i.e. predominant alignment of basal normals parallel to the direction of maximal stresses induced by phase transformation.

(4) By arc welding of cold-rolled sheets a wide spectrum of local conditions realizes within the TIZ, resulting in the formation of an extremely inhomogeneous structure. As the distance from the melting zone decreases, different processes become dominant in succession: recrystallization of deformed $\alpha$-grains without subsequent phase transformation, PT without preliminary recrystallization and PT after preliminary recrystallization. By intermediate regimes of local heat treatment each of these processes can take place only in grains with definite orientations.

\section{References}

Borodkina, M.M. and Spektor, E.N. (1981). X-ray Analysis of Texture in Metals and Alloys. Metallurgiya, Moscow, pp. 48-91.

Cheadle, B.A. and Ells, C.E. (1966). Electrochemical Technology, 4, 329-336.

Douglass, D.L. (1971). The Metallurgy of Zirconium. International Atomic Energy Agency, Vienna, pp. 6-232.

Isaenkova, M. and Perlovich, Yu. (1987). Fizika Metallov i Metallovedenie, 64, 107-112.

Isaenkova, M., Perlovich, Yu., Kapliy, S. and Shmelyova, T. (1988). Atomnaya Energiya, $65,42-45$.

Isaenkova, M., Perlovich, Yu., Shmelyova, T., Nikulina, A. and Zavyalov, A. (1989). Atomnaya Energiya, 67, 327-331.

Isaenkova, M., Perlovich, Yu. and Kapliy, S. (1994). Prediction of "stress-strain" curve for zirconium alloys: basic experimental facts and principles. In: Numerical Prediction of Deformation Processes and the Behaviour of Real Materials. Proceedings of the 15th Riso International Symposium on Material Science. Ed. by S.I. Andersen et al., Riso National Laboratory, Roskilde, Denmark, pp. 343-348. 
Perlovich, Yu. (1983). Inhomogeneity of strain hardening, recovery and recrystallization in molybdenum alloys with a developed crystallographic texture. In: Structure, Texture and Mechanical Properties of Deformed Molybdenum Alloys. Naukova Dumka, Kiev, pp. 87-145.

Perlovich, Yu. (1994). Development of strain hardening inhomogeneity during texture formation under rolling of bcc-metals. In: Numerical Prediction of Deformation Processes and The Behaviour of Real Materials. Proceedings of the 15th Riso International Symposium on Material Science. Ed. by S.I. Andersen et al., Riso National Laboratory, Roskilde, Denmark, pp. 445-450.

Tenckhoff, E. (1980). Verformungsmechanismen, Textur und Anisotropie in Zirkonium und Zircaloy. Mat.-techn. Reihe, N5, Gebruder Borntrager, Berlin-Stuttgart, $79 \mathrm{~S}$.

Zaymovskiy, A.S., Nikulina, A.V. and Reshetnikov, N.G. (1981). Zirconium Alloys in Atomic Energetics. Energoizdat, Moscow, pp. 13-212. 